Research Paper

\title{
Efficacy of Ahmed Glaucoma Valve Implantation on Neovascular Glaucoma
}

\author{
Zhan $\mathrm{Xie}^{1 *}$, Hai Liu²*, Mulong $\mathrm{Du}^{3}$, Min Zhu ${ }^{4}$, Sean Tighe ${ }^{5}$, Xue Chen ${ }^{1}$, Zhilan Yuan ${ }^{1}$ and Hong Sun ${ }^{\circledR}$ \\ 1. Department of Ophthalmology, Jiangsu Province Hospital, Nanjing, Jiangsu Province 210029, China \\ 2. Department of Ophthalmology, The Second People's Hospital of Yunnan Province (Fourth Affiliated Hospital of Kunming Medical University); Yunnan Eye \\ Institute; Key Laboratory of Yunnan Province for the Prevention and Treatment of ophthalmology (2017DG008); Provincial Innovation Team for Cataract \\ and Ocular Fundus Disease (2017HC010); Expert Workstation of Yao Ke (2017IC064), Kunming 650021, China \\ 3. Department of Biostatistics, School of Public Health, Nanjing Medical University, Nanjing, Jiangsu Province 210029, China \\ 4. Public Health, the University of Arizona, Tucson, Arizona, 85709, USA \\ 5. Tissue Tech, Inc., Ocular Surface Center, and Ocular Surface Research \& Education Foundation, Miami, FL 33173, USA \\ *The first two authors contribute equally to this work. \\ $\square$ Corresponding author: Hong Sun, M.D., Ph.D., Department of Ophthalmology, Jiangsu Province Hospital, 300 Guangzhou Road, Nanjing, Jiangsu Province \\ 210029, China; Telephone: +8613390766677; Fax: +86 (025) 68303160; Email: sunhong@njmu.edu.cn \\ (c) The author(s). This is an open access article distributed under the terms of the Creative Commons Attribution License (https://creativecommons.org/licenses/by/4.0/). \\ See http://ivyspring.com/terms for full terms and conditions.
}

Received: 2019.03.27; Accepted: 2019.08.02; Published: 2019.09.20

\begin{abstract}
To evaluate the efficacy of Ahmed glaucoma valve (AGV) implantation in treating neovascular glaucoma (NVG) and analyze the factors influencing the surgical success rate, a retrospective investigation of 59 NVG patients (66 eyes) who underwent AGV implantation was conducted at Jiangsu Province Hospital, China, from January 2014 to June 2018. Intraocular pressure (IOP), visual acuity, surgical success rates, medications, and complications were monitored at post-operative 1 day, 1 week, 1, 3, 6 and 12 months. Surgical success criteria were defined as $6 \mathrm{~mm} \mathrm{Hg}<1 O P<21$ $\mathrm{mmHg}$ with or without additional medications. Results showed average IOP was statistically significant between pre-operative visit and each follow-up visit (all $\mathrm{P}<0.05$ ). At 12 months, the success rate was $66.7 \%$. Multiple stepwise regression analysis suggested that age, panretinal photocoagulation (PRP), complications and hyphema were significant factors influencing the surgical success rate (all $P<0.05$ ). Thus, we conclude that AGV implantation is effective and safe for treatment of NVG. Surgical success is dependent on age, PRP, complications, and hyphema.
\end{abstract}

Key words: Ahmed glaucoma valve, influence factor, neovascular glaucoma, surgical success rate

\section{Introduction}

Glaucoma is a leading cause of irreversible blindness globally, which may affect up to 111 million people worldwide by 2040 [1-3]. Neovascular glaucoma (NVG), a severe secondary glaucoma, is closely related to retinal ischemic diseases. Ischemia triggers the release of various angiogenic factors, including vascular endothelial growth factor (VEGF), penetrating the anterior chamber to cause neovascularization of the iris and angle. Recently, with the increasing incidence of diabetes and vascular diseases, NVG is also increasing steadily, accounting for more than $30 \%$ of refractory glaucoma [4]. Therefore, selection of optimal treatments for NVG has become the focus to many ophthalmologists in clinics worldwide. Unfortunately, NVG patients usually respond poorly to anti-glaucoma drugs. Therefore, appropriate surgical interventions are usually required, including ciliary body destructive surgery, drainage valve implantation, and trabeculectomy with mitomycin C. Of these treatment options, drainage valve implantation has become increasingly popular for treatment of NVG patients due to the potential for severe complications in ciliary body destructive surgery and low success rates observed in conventional trabeculectomy surgery $[5,6]$.

Since 1993 when Ahmed glaucoma valves were first introduced in clinical practice, eye specialists 
around the world have gradually recognized their potential in the treatment of NVG $[7,8]$. The Ahmed valve has a one-way pressure-sensitive control valve, which restricts the drainage device to be operative only under an IOP of 8 to $14 \mathrm{mmHg}$, thus preventing early and late surgical complications, including excessive aqueous drainage, shallow anterior chamber and intraocular hypotension. Previously, Ahmed glaucoma valve (AGV) implantation was reserved for glaucoma patients poorly controlled after one or more filtration procedures, however recent evidence has recently encouraged its use as a primary surgery in refractory glaucoma such as secondary to neovascular glaucoma (NVG), pars plana vitrectomy (PPV), penetrating keratoplasty, and uveitis [9]. Unfortunately, the AGV has limitations of a small surface area and hypertensive phase [10-13] and its reported success rate varies greatly depending on the follow-up period and types of glaucoma [10, 14-16]. Importantly, effect of AGV on treatment of specific NVG has received little attention from the scientific world so far. Therefore, we have designed this study to evaluate the efficacy of AGV in treating NVG. Herein, we report the results of a retrospective study on 59 NVG patients (66 eyes) that underwent Ahmed glaucoma valve (AGV) implantation from January 2014 to June 2018. Such results may provide the scientific guidelines for clinicians to treat this disease effectively and successfully.

\section{Materials and Methods}

Data Sources We retrospectively reviewed the medical records of patients with NVG who underwent AGV implantation (model: FP7, New World Medical Inc., Rancho Cucamonga, CA, USA) at Jiangsu Province Hospital, China, between January 2014 and June 2018. This study was performed according to the Declaration of Helsinki and its subsequent revisions, and the ethics approval was obtained from Jiangsu Province Hospital Medical Ethics Committee.

Inclusion Criteria NVG patients were diagnosed clinically. NVG diagnostic criteria included typical iris neovascularization and ectropion uveae of pupillary margin, trabecular meshwork neovascularization, peripheral anterior synechia, increased IOP, decreased visual acuity, characteristic visual field defect, characteristic glaucomatous cup, and previous primary disease. Other inclusion criteria included: IOP maintained higher than $21 \mathrm{~mm} \mathrm{Hg}$ after applying IOP-lowering medications, post-operative follow-up period $>12$ months, and patient without severe systemic or mental diseases except primary glaucoma disease.
Exclusion criteria. Patients were excluded if they were $<14$ years old, had less than 12-month follow-up, or were in poor general physical conditions (such as unsatisfactory blood glucose control in diabetics).

Sample size: A total of 90 candidate patients were initially enrolled in this study. After review of inclusion and exclusion criteria, 66 patients $(73.3 \%)$ were enrolled for further analysis. Their basic information was summarized in Table 1.

\section{Table 1. Basic Data of Patients Enrolled}

\begin{tabular}{|c|c|}
\hline \multicolumn{2}{|l|}{ Characteristics } \\
\hline \multicolumn{2}{|l|}{ Gender } \\
\hline Male & $49(74.2 \%)$ \\
\hline Female & $17(25.8 \%)$ \\
\hline Age (Year) & $50.68 \pm 13.73$ \\
\hline \multicolumn{2}{|l|}{ Eye } \\
\hline Left-sided & $32(48.5 \%)$ \\
\hline Right-sided & $34(51.5 \%)$ \\
\hline Mean Course of disease (Month) & $9.27 \pm 14.43$ \\
\hline Mean Pre- BCVA & $2.01 \pm 1.34$ \\
\hline Mean Pre- IOP (mmHg) & $39.30 \pm 12.19$ \\
\hline Mean Pre-medications No. & $2.58 \pm 0.56$ \\
\hline Mean 1-year post-medication No. & $1.03 \pm 1.05$ \\
\hline PRP history & $40(60.6 \%)$ \\
\hline Pre-anti-VEGF history & $31(47.0 \%)$ \\
\hline \multicolumn{2}{|l|}{ Lens status } \\
\hline Phakic & $44(66.7 \%)$ \\
\hline Pseudophakic & $18(27.3 \%)$ \\
\hline Aphakic & $4(6.1 \%)$ \\
\hline \multicolumn{2}{|l|}{ Primary Diseases } \\
\hline CRVO+BRVO & $7(10.6 \%)$ \\
\hline $\mathrm{DM}$ & $41(62.1 \%)$ \\
\hline PACG & $4(6.1 \%)$ \\
\hline Trauma & $5(7.6 \%)$ \\
\hline \multicolumn{2}{|l|}{ Previous History } \\
\hline PPV & $8(12.1 \%)$ \\
\hline Cataract surgery & $13(19.7 \%)$ \\
\hline Trabeculectomy & $1(1.5 \%)$ \\
\hline Transscleral cyclophotoculation & $2(3.0 \%)$ \\
\hline
\end{tabular}

Surgical Method All surgeries were completed by the same experienced glaucoma specialist. The surgical procedure was as follows:(1) $2.5 \mathrm{ml}$ of $2 \%$ lidocaine $+0.75 \%$ bupivacaine (mixed at a ratio of 1:1) was applied for peribulbar anesthesia. (2) With a suspension wire made in the corneal limbus, the bulbar conjunctiva above the temple was cut along the corneal limbus, and a fornix-based conjunctival flab was created to expose 2 recti muscles. A pocket was performed between the episclera and tenon's capsule by blunt dissection. After the fascia was separated, hemostasis was drained by cautery. A $4 \times 4 \mathrm{~mm}$ sclera flap with a $50 \%$ scleral thickness was made above the temple. (3) The tube of the valve was irrigated with balanced saline solution to open the valve mechanism. Then the Ahmed valve was inserted between the lateral rectus and superior rectus, and a 6-0 suture 
was fastened at $10 \mathrm{~mm}$ behind the corneal limbus. (4) A lateral corneal incision was made, sodium hyaluronate was injected into the anterior chamber, and an anterior chamber puncture was made in the corneal limbus under the sclera flap. The drainage tube was inserted into the anterior chamber from the puncture site. (5) The sclera flap was closed with 10-0 suture, the drainage tube was fixed and partly ligated for 1 stitch with 8-0 absorbable suture, and the conjunctival flap was sutured.

Evaluation criteria of surgical efficacy NVG patients were usually accompanied by diseases of the ocular fundus, such as diabetic retinopathy (DM) and central retinal vein occlusion (CRVO), which have led to poor visual performance and affected examinations of the visual acuity, visual field and OCT. Therefore, visual acuity, cup-disc ratio, visual field, and retinal nerve fiber layer thickness may not be treated as the criteria of surgical success. In this research, IOP served as the major indicator for evaluating surgical success or failure.

Evaluation of Surgical Success or Failure (1) Success: IOP was 6 to $21 \mathrm{mmHg}$ without antiglaucoma medications post-operatively.

Failure: IOP was lower than $6 \mathrm{mmHg}$ or higher than $21 \mathrm{mmHg}$ after applying antiglaucoma medications post-operatively. In such cases, severe eye complications were observed, for example, retinal detachment and endophthalmitis.

Follow-up Period Regular follows-ups were conducted at 1 day, 1 week, 1, 3, 6, and 12 months after surgery.

Observational Indexes Best corrected visual acuity (BCVA) was tested by an optometrist using a standard logarithmic visual acuity chart (Yuehua Medical Apparatus and Instruments, Inc., Shantou, Guangdong, China) at 5 meters. The observational indexes included best corrected visual acuity (BCVA, Standard Logarithmic Visual Acuity E Chart), preoperative and post-operative IOP (Goldmann applanation Tonometer, Hagg-Streit, Switzerland), slit-lamp (BM900, Switzerland) microscopic examination, type and number of local and systemic applications of antiglaucoma medications and postoperative complications.

Statistical analysis We have enrolled all available patients for analysis and compared multiple levels about clinical efficacy, including the IOP in pre vs. pro-surgery, and stepwise regression analysis for surgical success rate at 1-year follow-up, depending on the research purposes. All statistical analyses were conducted using the SPSS 21.0. For continuous variables, t-test or paired t-test was performed for data with normal distribution, while a corresponding non-parametric test was used for abnormal distribution data. For categorical variables, Pearson's chi-square test or Fisher's exact test was used. A multiple stepwise regression analysis was used for the effect evaluation of the candidate risk factors. A p-value of less than 0.05 was considered statistically significant.

\section{Results}

The mean pre-operative IOP was $48.23 \pm 8.17$ $\mathrm{mmHg}$. At 1 day, 7 days, and 1,3, 6, and 12 months after AGV implantation, the mean IOP was $16.70 \pm 10.79, \quad 14.19 \pm 6.03, \quad 19.03 \pm 8.00, \quad 19.43 \pm 5.59$, $20.31 \pm 5.96$ and $21.68 \pm 6.64 \mathrm{mmHg}$ respectively. The difference between the mean baseline IOP and the IOP at each follow-up point was statistically significant $(\mathrm{P}<0.05$, Figure 1$)$.

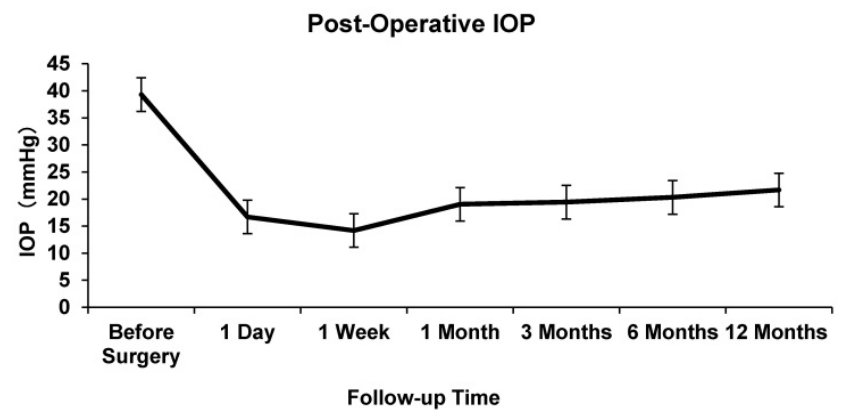

Figure 1. Intraocular Pressure at Baseline and Follow-up

A significant decrease in BCVA at 1 year post-operatively was noted $(\mathrm{P}<0.05)$ compared with the baseline BCVA. The success rate in the entire study population was $66.7 \%$ at 1 year after the operation. An average of $2.58 \pm 0.56$ antiglaucoma medications were applied in all patients pre-operatively, while an average of $1.03 \pm 1.05$ medications was applied post-operatively at the last follow-up, which was statistically significant $(\mathrm{P}<0.001)$.

The most common post-operative complication was hyphema, which could be seen in 10 eyes (15.2\%). Nine eyes developed shallow anterior chamber $(13.6 \%)$. Two eyes had malignant glaucoma $(3 \%)$. Other post-operative complications included corneal endothelial decompensation (1.5\%), choroidal detachment $(1.5 \%)$, drainage valve exposure $(1.5 \%)$, low tension retinopathy $(1.5 \%)$ and drainage valve displacement (1.5\%) (Table 2).

Single factor analysis for failure was performed. Factors such as age, gender, course of disease, PRP history, primary disease, lens status, pre-operative anti-VEGF therapy, pre-operative IOP, pre-operative BCVA, pre-medications number, previous operation history and surgical complications were included. A multiple stepwise regression analysis ascertained the 
relative predictive ability of these factors. Our results suggest that age, PRP, no post-operative complications and post-operative hyphema are statistically-significant factors influencing the surgical success rate (Table 3 ).

Table 2. Post-operative Complication Rates

\begin{tabular}{ll}
\hline Complication & $\mathrm{N}(\%)$ \\
\hline Hyphemia & $10(15.2 \%)$ \\
Shallow anterior chamber & $9(13.6 \%)$ \\
Choroidal detachment & $1(1.5 \%)$ \\
Corneal decompensation & $1(1.5 \%)$ \\
Hypotony maculopathy & $1(1.5 \%)$ \\
Drainage tube exposure & $1(1.5 \%)$ \\
Drainage tube displacement & $1(1.5 \%)$ \\
Malignant glaucoma & $2(3 \%)$ \\
\hline
\end{tabular}

Table 3. Stepwise Regression Analysis of Factors Influencing Surgical Success Rate at 1 Year after Surgery

\begin{tabular}{lllllll}
\hline Factors & B & S.E. & Wald & DOF & P & Exp (B) \\
\hline Age & -0.083 & 0.031 & 7.296 & 1 & 0.007 & 0.92 \\
No post-operative complication & & & 9.443 & 3 & 0.024 & \\
Hyphema & 4.622 & 1.524 & 9.205 & 1 & 0.002 & 101.735 \\
Shallow anterior chamber & 0.631 & 1.151 & 0.301 & 1 & 0.583 & 1.88 \\
Other & 0.304 & 0.874 & 0.121 & 1 & 0.728 & 1.355 \\
PRP history & 2.574 & 0.858 & 8.996 & 1 & 0.003 & 13.117 \\
\hline B, the slope of the line; S.E., standard error; Wald, Wald test; DOF, degree of \\
freedom; P, p-value; Exp (B), odds ratio.
\end{tabular}

\section{Discussion}

Previously, Yalvac et al reported the 1-year surgical success rate of $38 \mathrm{NVG}$ eyes receiving Ahmed valve implantation was $63.3 \%$ [17] and Netland reported a 1-year surgical success rate of $73.1 \%$ in 38 eyes [18]. Similarly, our results showed a success rate of $66.7 \%$ at 12 months in Asian patients.

Risk factors for effectiveness of glaucoma valve implantation are still unclear. Sidoti et al performed Baerveldt drainage valve implantation in $36 \mathrm{NVG}$ patients and discovered that being young was a risk factor of surgical failure [19]. Tsai et al [20] and Takihara et al [6] performed filtering surgery on NVG patients with a similar conclusion. Mermoud et al discovered in their research that NVG patients aged over 55 years had a higher Molteno surgical success rate than those aged below 55 years [21]. Based on these data and our current results, age is an important factor influencing surgical success rate. This may be linked to the stronger wound healing response in younger patients, making them more likely to develop fiber-wrap in the periphery of the drainage disc, as well as more aggressive illness when the younger patients develop NVG. Therefore, we suggest that clinicians should strengthen the disease education for young NVG patients, make pre-operative disease evaluation, and optimize the treatment plan and follow up after treatment.

Hamard et al believed that for NVG, doctors should not only treat the primary disease, but also improve the pathological status of retinal ischemia [22]. One potential way is PRP, which ablates the ischemic retina to decrease tissue oxygen demand, thus reducing VEGF release and the formation of NV. Evans et al found that neo-vessels of iris disappeared in $68 \%$ of NVG patients after sufficient retinal photocoagulation. In this study, we found that retinal photocoagulation history was a factor influencing the success rate of surgery 1 year after AGV in NVG patients $(\mathrm{P}<0.01)$. Patients who did not receive retinal photocoagulation therapy were more likely to fail after the implantation of Ahmed valve. Therefore, we believe that clinicians should timely conduct standardized retinal photocoagulation after implantation of Ahmed valve drainage for NVG patients. Based on the treatment of primary diseases leading to NVG, standardized retinal photocoagulation is the fundamental method to promote retinal angiogenesis regression and inhibit the growth of retinal angiogenesis.

Another potential risk factor is hyphema. In the research by Choo J et al, postoperative hyphema could be associated with increased conjunctival inflammation and scarring, leading to a higher risk of failure [23]. Shunji Nakatake et al discovered in their research that post-operative hyphema increased concentrations of some cytokines may lead to a failure of conjunctival bleb formation [24]. Our research confirmed incidence of post-operative hyphema is a risk factor for failure, resembling what has previously reported.

Recently, the intravitreal injection of anti-VEGF drugs has shown promising results in regression of neovascularization [25-27]. Adjuvant anti-VEGF treatment may lead to regression of $\mathrm{NV}$ in the iris and angle, thus, reducing the incidence of hyphema, providing time and conditions for the subsequent panretinal photocoagulation, therefore, potentially enhancing the surgical outcome of AGV implantation in NVG $[6,28]$. However the effect of anti-VEGF drug may be transient, hence repeated injections are sometimes required. Additionally, Kwon et al demonstrated pre-operative injection of anti-VEGF drugs increases the success rate of AGV implantation when peripheral anterior synechiae is less than $1 / 2$ [29]. Our study concluded similarly that preoperative anti-VEGF therapy was not associated with a better surgical success at 1 year after drainage valve implantation. This may be due to regression of iris neovascularization which may persist for 8-10 weeks after intraocular injection but then return to its previous condition within 6 months after 
administration, thus playing a limited, temporary role in the treatment of NVG. Most patients in this study had advanced NVG, which may be one of the reasons why anti-VEGF treatment showed no significant therapeutic effect. Therefore, further studies are needed to determine whether anti-VEGF therapy shows a positive effect on the surgical outcome of NVG, particularly during the early stages. In addition, there is often a selection bias in patients receiving anti-VEGF drug therapy at present. In general, patients with more severe diseases are recommended to receive anti-VEGF drug therapy.

Different opinions concerning whether primary diseases in patients will affect surgical effects are noted in the present study. Every et al discovered in their research that the surgical success rate between CRVO patients receiving drainage valve implantation and those without CRVO was comparable [30]. Nevertheless, the success rate is related to the progress of primary vascular diseases. Mermoud et al suggested that surgical effects in diabetics were superior to those in central retinal vein occlusion (CRVO) patients [21]. Hayreh considered that patients with CRVO as the primary disease generally had more severe illness than diabetics and NVG patients, thus, affecting the surgical outcome and prognosis [4]. In the study of $\mathrm{Ye} \mathrm{H}$, the difference in the surgical success rates among CRVO patients, diabetics, and patients with other diseases was not statistically significant [31]. Similarly, our results show no statistical differences related to other diseases in our study. However, we cannot make definitive conclusions due to sample size.

Finally, the absence of postoperative complications was an influencing factor for the success rate at 1 year $(\mathrm{P}<0.05)$. Postoperative complications after AGV may include postoperative anterior chamber hyphema, shallow anterior chamber, malignant glaucoma, choroidal detachment, corneal endothelial decompensation, drainage valve exposure, and drainage valve displacement. In our study, the shallow AC rate was 9\%, due to over aqueous humor filtration. Our research showed that postoperative low-tension shallow anterior chamber overwhelmingly belonged to grade I according to the 3-grade classification, which could be cured with conservative treatment. Although it was the second most common complication after AGV in our study, we cannot conclude that the occurrence of shallow AC was related to surgical success rate.

In summary, AGV implantation is effective and safe for treatment of NVG. Age, standardized retinal photocoagulation history, no postoperative complications and postoperative anterior chamber hyphema are influencing risk factors for the success of
AGV surgery. Careful operation should be performed during the operation, complications should be avoided, and standardized retinal photocoagulation should be performed in time after the operation.

\section{Competing Interests}

The authors have declared that no competing interest exists.

\section{References}

1. Quigley HA, Broman AT. The number of people with glaucoma worldwide in 2010 and 2020. BrJOphthalmol. 2006; 90: 262-7.

2. Jonas JB, Yang D, Wang N. [Effect of intraocular pressure on glaucomatous damage to the optic nerve]. Ophthalmologe. 2014; 111: 181-8; quiz 9-90.

3. Tham YC, Li X, Wong TY, Quigley HA, Aung T, Cheng CY. Global prevalence of glaucoma and projections of glaucoma burden through 2040: a systematic review and meta-analysis. Ophthalmology. 2014; 121: 2081-90.

4. Hayreh SS. Neovascular glaucoma. Prog Retin Eye Res. 2007; 26: 470-85.

5. SooHoo JR, Seibold LK, Kahook MY. Recent advances in the management of neovascular glaucoma. Semin Ophthalmol. 2013; 28: 165-72.

6. Takihara $\mathrm{Y}$, Inatani $\mathrm{M}$, Fukushima $\mathrm{M}$, Iwao $\mathrm{K}$, Iwao $\mathrm{M}$, Tanihara $\mathrm{H}$. Trabeculectomy with mitomycin $\mathrm{C}$ for neovascular glaucoma: prognostic factors for surgical failure. Am J Ophthalmol. 2009; 147: 912-8, 8 e1.

7. Gedde SJ, Singh K, Schiffman JC, Feuer WJ, Tube Versus Trabeculectomy Study G. The Tube Versus Trabeculectomy Study: interpretation of results and application to clinical practice. Curr Opin Ophthalmol. 2012; 23: 118-26.

8. Zhou M, Wang J, Sun X. Efficacy and safety of intravitreal bevacizumab in eyes with neovascular glaucoma undergoing ahmed glaucoma valve implantation: 2-year follow-up. Acta Ophthalmol. 2016; 94: e78.

9. Desai MA, Gedde SJ, Feuer WJ, Shi W, Chen PP, Parrish RK, 2nd. Practice preferences for glaucoma surgery: a survey of the American Glaucoma Society in 2008. Ophthalmic Surg Lasers Imaging. 2011; 42: 202-8.

10. Pandav SS, Seth NG, Thattaruthody F, Kaur M, Akella M, Vats A, et al. Long-term outcome of low-cost glaucoma drainage device (Aurolab aqueous drainage implant) compared with Ahmed glaucoma valve. Br J Ophthalmol. 2019.

11. Pakravan M, Rad SS, Yazdani S, Ghahari E, Yaseri M. Effect of early treatment with aqueous suppressants on Ahmed glaucoma valve implantation outcomes. Ophthalmology. 2014; 121: 1693-8.

12. Law SK, Kornmann HL, Giaconi JA, Kwong A, Tran E, Caprioli J. Early Aqueous Suppressant Therapy on Hypertensive Phase Following Glaucoma Drainage Device Procedure: A Randomized Prospective Trial. J Glaucoma. 2016; 25: 248-57.

13. Riva I, Roberti G, Katsanos A, Oddone F, Quaranta L. A Review of the Ahmed Glaucoma Valve Implant and Comparison with Other Surgical Operations. Adv Ther. 2017; 34: 834-47.

14. Pathak Ray V, Rao DP. Surgical outcomes of a new affordable non-valved glaucoma drainage device and Ahmed glaucoma valve: comparison in the first year. Br J Ophthalmol. 2018.

15. Souza C, Tran DH, Loman J, Law SK, Coleman AL, Caprioli J. Long-term outcomes of Ahmed glaucoma valve implantation in refractory glaucomas. AmJOphthalmol. 2007; 144: 893-900.

16. Bouhenni R, Krasniqi M, Dunmire J, Lagouros E, Woodruff T, Bates J, et al. Long-term Outcomes of Baerveldt Glaucoma Implant Shunts as a Primary Versus Secondary Procedure. J Glaucoma. 2018; 27: 1169-74.

17. Yalvac IS, Eksioglu U, Satana B, Duman S. Long-term results of Ahmed glaucoma valve and Molteno implant in neovascular glaucoma. Eye (Lond). 2007; 21: 65-70.

18. Netland PA. The Ahmed glaucoma valve in neovascular glaucoma (An AOS Thesis). Trans Am Ophthalmol Soc. 2009; 107: 325-42.

19. Sidoti PA, Dunphy TR, Baerveldt G, LaBree L, Minckler DS, Lee PP, et al. Experience with the Baerveldt glaucoma implant in treating neovascular glaucoma. Ophthalmology. 1995; 102: 1107-18.

20. Tsai JC, Feuer WJ, Parrish RK, 2nd, Grajewski AL, 5-Fluorouracil filtering surgery and neovascular glaucoma. Long-term follow-up of the original pilot study. Ophthalmology. 1995; 102: 887-92; discussion 92-3.

21. Mermoud A, Salmon JF, Alexander P, Straker C, Murray AD. Molteno tube implantation for neovascular glaucoma. Long-term results and factors influencing the outcome. Ophthalmology. 1993; 100: 897-902.

22. Hamard P, Baudouin C. [Consensus on neovascular glaucoma]. J Fr Ophtalmol. 2000; 23: 289-94.

23. Evans JR, Michelessi M, Virgili G. Laser photocoagulation for proliferative diabetic retinopathy. Cochrane Database Syst Rev. 2014: CD011234.

24. Nakatake S, Yoshida S, Nakao S, Arita R, Yasuda M, Kita T, et al. Hyphema is a risk factor for failure of trabeculectomy in neovascular glaucoma: a retrospective analysis. BMC Ophthalmol. 2014; 14: 55

25. Kang JY, Nam KY, Lee SJ, Lee SU. The effect of intravitreal bevacizumab injection before Ahmed valve implantation in patients with neovascular glaucoma. Int Ophthalmol. 2014; 34: 793-9. 
26. Zhou MW, Wang W, Huang WB, Chen SD, Li XY, Gao XB, et al. Adjunctive with versus without intravitreal bevacizumab injection before Ahmed glaucoma valve implantation in the treatment of neovascular glaucoma. Chin Med J (Engl). 2013; 126: 1412-7.

27. Li Z, Zhou M, Wang W, Huang W, Chen S, Li X, et al. A prospective comparative study on neovascular glaucoma and non-neovascular refractory glaucoma following Ahmed glaucoma valve implantation. Chin Med J (Engl). 2014; 127: 1417-22.

28. Tsai JC, Johnson CC, Dietrich MS. The Ahmed shunt versus the Baerveldt shunt for refractory glaucoma: a single-surgeon comparison of outcome. Ophthalmology. 2003; 110: 1814-21.

29. Kwon J, Sung KR. Effect of Preoperative Intravitreal Bevacizumab on the Surgical Outcome of Neovascular Glaucoma at Different Stages. J Ophthalmol. 2017; 2017: 7672485.

30. Every SG, Molteno AC, Bevin TH, Herbison P. Long-term results of Molteno implant insertion in cases of neovascular glaucoma. Arch Ophthalmol. 2006; 124: $355-60$.

31. He Y, Tian Y, Song W, Su T, Jiang H, Xia X. Clinical efficacy analysis of Ahmed glaucoma valve implantation in neovascular glaucoma and influencing factors: A STROBE-compliant article. Medicine (Baltimore). 2017; 96: e8350. 